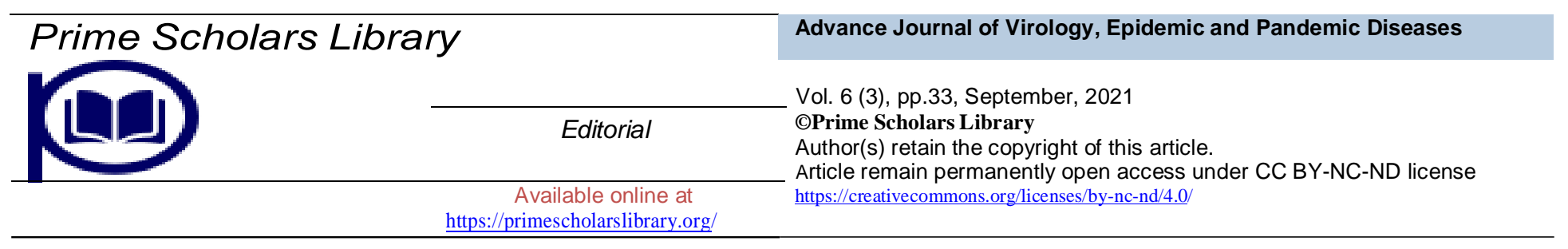

\title{
Emergent virus and zoonosis
}

\author{
Welburn Nakao* \\ Department of Disease Control, Hokkaido University, Sapporo, \\ Japan.
}

\section{INTRODUCTION}

An emerging virus is a virus that has a significant increase in incidence/geographical scope or it will have the potential to increase in the near future. Emerging viruses are one of the main causes of emerging infectious diseases and cause public health problems on a global scale because they may cause disease outbreaks, leading to epidemics and pandemics. In addition to causing disease, emerging viruses can also have serious economic impacts. Recent examples include the SARS-related coronavirus, which caused the SARS 20022004 (SARSCoV1) outbreak and the COVID19 SARSCoV2 pandemic 2019- 21.

Other examples include the human immunodeficiency virus that causes HIV/AIDS; the virus that causes Ebola; the H5N1 influenza virus that causes avian influenza; and H1N1/09 caused the 2009 swine flu pandemic, while the earlier H1N1 strain caused The 1918 Spanish flu pandemic. The appearance of viruses in humans is usually the result of zoonotic diseases, which involve the transfer of viral diseases from other animals to humans between species. Due to the presence of zoonotic viruses in animal reservoirs, it is much more difficult to eradicate them, so persistent infections can be established in thepopulation.

Recently emerging viruses should not be confused with newly emerging viruses or newly detected viruses. Viruses that have come back are often thought of as returning viruses, such as measles. The newly detected virus is a previously unrecognized virus that has spread in the species as an endemic or epidemic infection. Newly detected viruses may not be classified because they do not leave obvious clues and/or they cannot be isolated or reproduced in cell culture. Examples include human rhinovirus the leading cause of the common cold first discovered in 1956, hepatitis $C$, and human metapneumovirus. Since the detection of such viruses is driven by technology, the number of reports may increase.

\section{ZOONOSIS}

The most common cause of emerging viruses in humans is zoonotic diseases. Zoonotic diseases are caused by harmful bacteria such as viruses, bacteria, parasites and fungi. These bacteria can cause many different types of diseases in humans and animals, ranging from mild to severe disease and even death.

\section{PREVENTION OF ZOONOSIS}

The control and prevention of zoonotic diseases depend on adequate global surveillance at all levels, including the identification of new pathogens, public health surveillance, including serological investigations, and analysis of the risks of transmission. The complexity of zoonotic events around the world heralds a multidisciplinary approach to prevention. A health model has been proposed as a global strategy to help prevent the occurrence of zoonotic diseases in humans, including new viral diseases. The One Health concept aims to promote different related disciplines, including wildlife biology, veterinary medicine, medicine, agriculture, ecology, microbiology, epidemiology, and biomedical engineering. 